\title{
Hybrid Modeling of Materials Properties for Improved CAE-Simulations
}

\author{
Uwe Diekmann ${ }^{1, a^{*}}$, Alex Miron ${ }^{1, b}$, Andreea Trasca ${ }^{1, c}$ \\ ${ }^{1}$ Metatech GmbH, Lünener Str. 211/212, D-59174 Kamen, Germany \\ auwe.diekmann@metatech.pro, balex.miron@metatech.pro, candreea.trasca@metatech.pro
}

Keywords: Flow stress analysis, mathematical modelling, curve fitting, visualization

\begin{abstract}
The new MatPlus software supports the multi-dimensional modelling of materials properties using different data sources. Extensive mathematical functions allow curve fitting of data from different sources to any constitutive models and selectively combining models and datapoints along different dimensions. Physically consistent extrapolation of measured data within the complete multi-dimensional parametric space can be achieved. An integrated library of models can be extended by the user and already contains many popular equations like Hensel-Spittel and Zerilli-Armstrong for flow curves.
\end{abstract}

\section{Introduction}

Modeling of materials properties is complicated due of many reasons. Measurements as function of temperature, temperature gradients, phase transformations are time consuming and expensive even for a single chemical composition, statistical validation is therefore not always done. Due to the interplay of multiple, often unknown effects and experimental environments it is frequently impossible to consolidate measured data from different sources in a straightforward fashion. Therefore, simulation is a vital part in process and materials optimization, promising to offer consistent results over wide range of materials and parameters. Widely used commercial packages like JMatPro [1] use macroscopic/thermodynamic models fitted across specific alloy classes.

However, generic models, while correctly predicting variations along some coordinates like temperature, show discrepancies in areas where local microstructure effects are important, like in modeling stress vs. strain flow properties. A reasonable approach is then to extract the 'good' information from the simulation tool and use it to correct or expand measured data.

Recently a hybrid modeling environment - MatPlus - was developed, where such a task can be easily achieved. While being, at its core, a high-performance database for scientific / technical multi-dimensional data, the system offers specific tools for interacting with JMatPro (or other) simulation software or loading measurement data in various formats. Freely definable mathematical functions allow multi-dimensional fitting of data from different sources to complex mathematical models and selectively combining models and data points along different dimensions. Physically consistent extrapolation of measured data within the complete multi-dimensional parametric space can be achieved. An integrated library of models can be extended by the user and already contains many popular equations like Hensel-Spittel [2] for flow curves.

In this paper an example of how to generate a synthetic set of flow curves from JMatPro flow stress calculations coupled to measured data using the MatPlus system is shown.

\section{The Flow Grid Model}

In a minimalistic view, the stress $\sigma$ is a function of strain $\varphi$, strain rate $\dot{\varphi}$ and temperature $T$ : $\sigma(\varphi, \dot{\varphi}, T)$. Albeit a function over a continuous three-dimensional space, it is usually measured (and computed) as a set of $\sigma(\varphi)$ curves at some discrete values of $\dot{\varphi}, T$.

Usually there are significant differences in measured flow curves coming from various experiments, as well as differences between experimental data and simulated flow curves. More often than not, far fewer measurements points are available than needed to accurately probe the dependence of $\sigma$ on $\dot{\varphi}, T$. Therefore, as a starting point, the stress function was decomposed as follows:

$$
\sigma(\varphi, \dot{\varphi}, T)=F\left(\varphi, \dot{\varphi}_{0}, T_{0}\right) G(\dot{\varphi}, \varphi) E(T, \varphi)
$$


where $F(\varphi)$ is interpolated from a few credible (and overlapping) measurements and simulation points at some specific $\dot{\varphi}_{0}$ and $T_{0}$. G,E are empirical functions fitted to a larger set of measurements and simulation, where large discrepancies in absolute values are counterbalanced by a similar trend along $\dot{\varphi}$ and $T$ respectively. $G, E$ have a value of 1 at $\left(\dot{\varphi}_{0}, T_{0}\right)$. In other words, the useful information from different sets of data and combine individual $2 \mathrm{D}$ models into a multidimensional function were extracted.

\section{Generation of Synthetic Sets of Flow Curves}

To illustrate the method data $(\sigma, \varphi)$ for a 22MnB5 alloy from Hochholdinger [3], Naderi [4] and JMatPro [1] calculations were considered. Since the flow curves from the three sources show significant differences in both absolute values and shape, a direct global fit of all of them would give poor results. The procedure is the following: in the $(\sigma, \varphi)$ view a reference curve for a chosen strain rate $\dot{\varphi}_{0}$ and temperature $T_{0}$ was fitted. Using then the $(\sigma, \dot{\varphi})$ and $(\sigma, T)$ views, the variation along the other coordinates was modeled separately.

Stress-Strain View. Thus in a first step the strain rate and temperature conditions were chosen from the various sources, where the flow curves are most congruous, and fit those to an empirical model. Here the reference curves at $T_{0}=800^{\circ} \mathrm{C}$ and $\dot{\varphi}_{0}=1 / \mathrm{s}$ were chosen, and as fitting function

$$
f(\varphi)=d\left[1-\beta \exp \left(-M \varphi^{n}\right)\right]\left[1+\alpha \exp \left(-c\left(\varphi-\varphi_{0}\right)^{2}\right)\right]
$$

was used, which consists of a Hocket-Sherby hardening term (the first square bracket) and a term which corresponds to softening effects (the second square bracket). The chosen flow curves from different sources and the fitted reference curve are shown in Fig. 1 (left). Note that JMatPro predicts an abrupt softening transition which is usually not reflected in the measurements. This is due to the fact that JMatPro uses two separate stress models corresponding to the hardening and softening parts of the flow curves, and the two models are just connected at the point where the kink appears. By optional weighting of the individual curves, our environment also allows directed fitting towards a biased optimum (here simple non-biased optimization was used). Our reference curve then softly exhibits the behavior predicted by JMatPro, while mainly adhering to the experimental values.
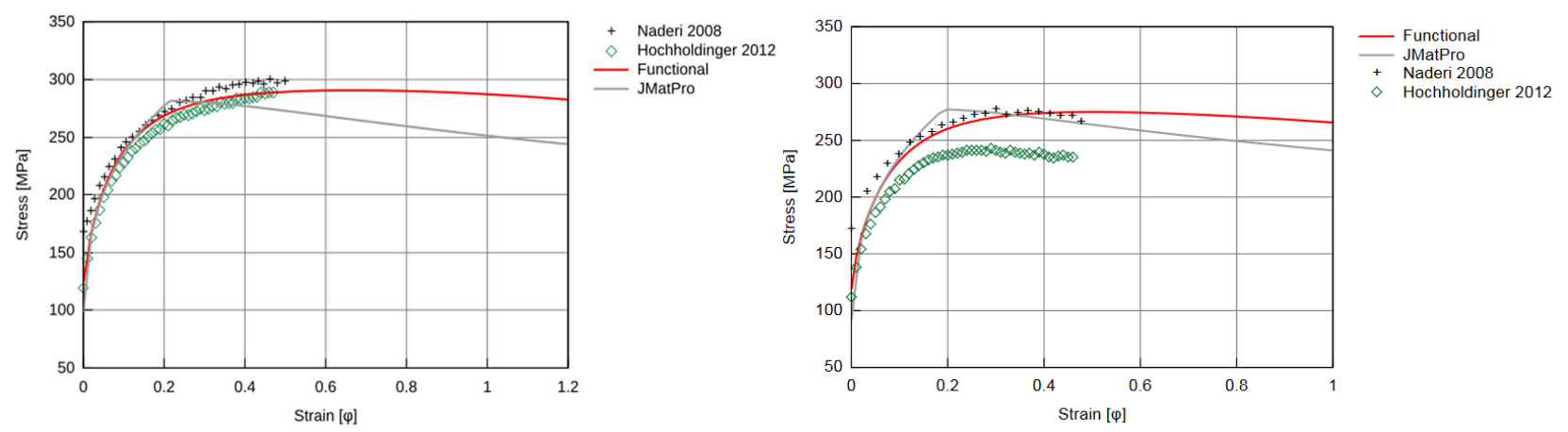

Fig.1. Left: Fitted (reference) curve to matching flow curves from literature and JMatPro for $22 \mathrm{MnB} 5$ at $T_{0}=800^{\circ} \mathrm{C}$ and $\dot{\varphi}_{0}=1 / \mathrm{s}$. Right: functional expansion at $T=750^{\circ} \mathrm{C}$ and $\dot{\varphi}=0.11 / \mathrm{s}$.

Next the other remaining dimensions of the stress function, namely the strain rate and temperature variations, are incorporated. To be able to convert the measured $(\sigma, \varphi)$ flow curves at different strain rates and temperatures in $(\sigma, \dot{\varphi})$ and $(\sigma, T)$ curves, each $(\sigma, \varphi)$ curve was resampled so that data points can be generated at the same consistent strain values. This enables to build views of the flow curves as a function of strain rate or temperature.

Stress-Temperature View. It is known that as the temperature increases, the stress in the material decreases. Following other models (e.g. Hensel-Spittel [2]), the stress decays exponentially with temperature was considered. Thus in the $(\sigma, T)$ view various flow stress curves are fitted by an exponential function: $G(T)=a \exp (-b T)$ and the strain rate variation is at first neglected. The parameter $b$ will depend on $\varphi$ as shown later. Fig. 2 shows such an exponential fit at $h i=0.4$. 


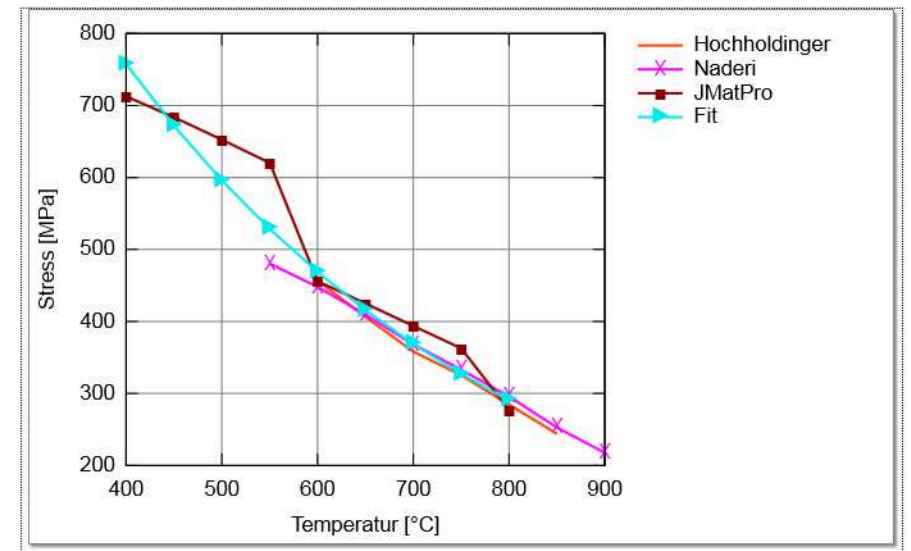

Fig. 2. Exponential fit for $\sigma, T$ data at $\varphi=0.4$.

Note the characteristic discontinuous jump predicted by JMatPro, characteristic of a phase/mechanism transition. For simplicity, and lacking experimental data to confirm this behavior, a simpler model while still accounting was chosen, coarsely, for the JMatPro data points. Since absolute errors among the data points are not of interest, the parameter $a$ (and /or shift the curves to aid in the fitting of the decay factor $b$ ) was neglected. This exponential fitting can be done for every value of strain, thus $b$ as a function of $\varphi$ can be found. Examining this variation shown in Fig. 3 it was decided to express it as a power-function

$$
b(\varphi)=m_{1} \varphi^{m_{2}}+m_{3}
$$

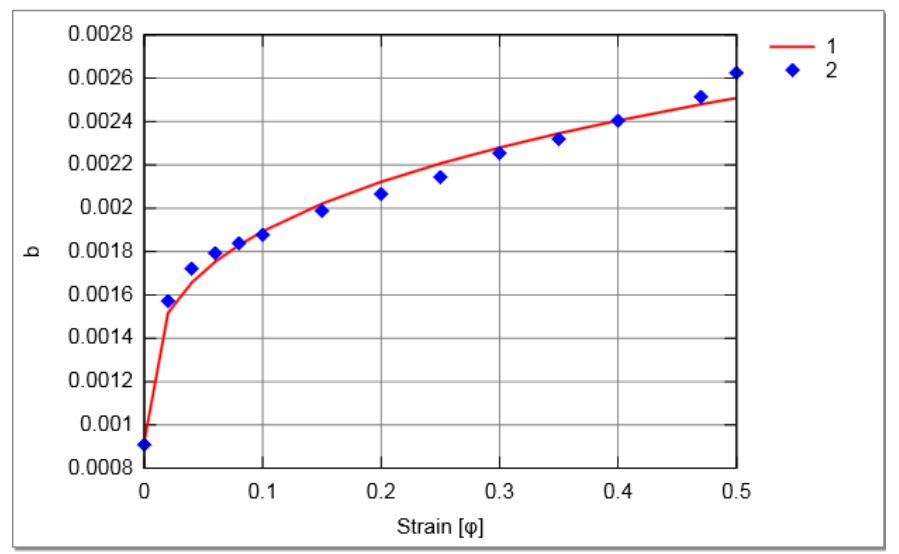

Fig.3. Fitting of $b$ Parameter as a function of $\varphi$

Stress-Strain Rate View. Analogously, the variation of stress with strain rate can be modeled using a power function $(\dot{\varphi})^{d}$. Similar to the $(\sigma, T)$ view, here the $(\sigma, \dot{\varphi})$ curves for each strain $\varphi$ and temperature $T$ were fitted and then express parameter $d$ as a function of strain $\varphi: d=p_{1} \varphi^{p_{2}}+p_{3}$. Fig4. a) and b) show a stress-strain rate fit and the fitting of the parameter $d$.
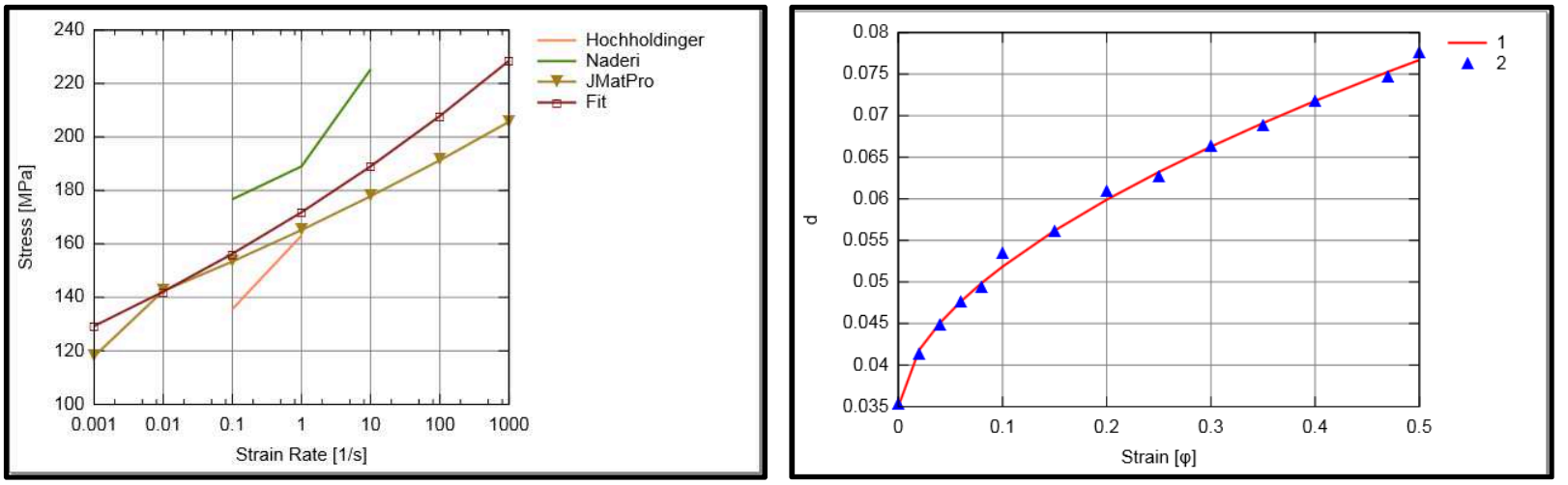

Fig.4. a) Fit in $(\sigma, \dot{\varphi})$ view for a specific strain and temperature, b) Variation of the parameter $d$ with strain $\varphi$. 
Expansion of the Reference Curve over all Variables. Knowing the variation of the stress function over all variables, the complete form of the stress functional can be written:

$$
\begin{aligned}
& \sigma=d\left[1-\beta \exp \left(-M \varphi^{n}\right)\right]\left[1+\alpha \exp \left(-c\left(\varphi-\varphi_{0}\right)^{2}\right)\right] \exp \left[-\left(m_{1} \varphi^{m_{2}}+m_{3}\right)(T-\right. \\
& \left.\left.T_{0}\right)\right](\dot{\varphi})^{\left(p_{1} \varphi^{p_{2}}+p_{3}\right)}
\end{aligned}
$$

Here are all parameters known from the previous calculations and now flow curves for any strain, strain rate and temperature can bei plotted. Fig. 5 shows the generated set of flow curves for $22 \mathrm{MnB} 5$ for strain rate $1 / \mathrm{s}$ and various temperatures, a comparison with source data is shown in Fig. 1 (right).

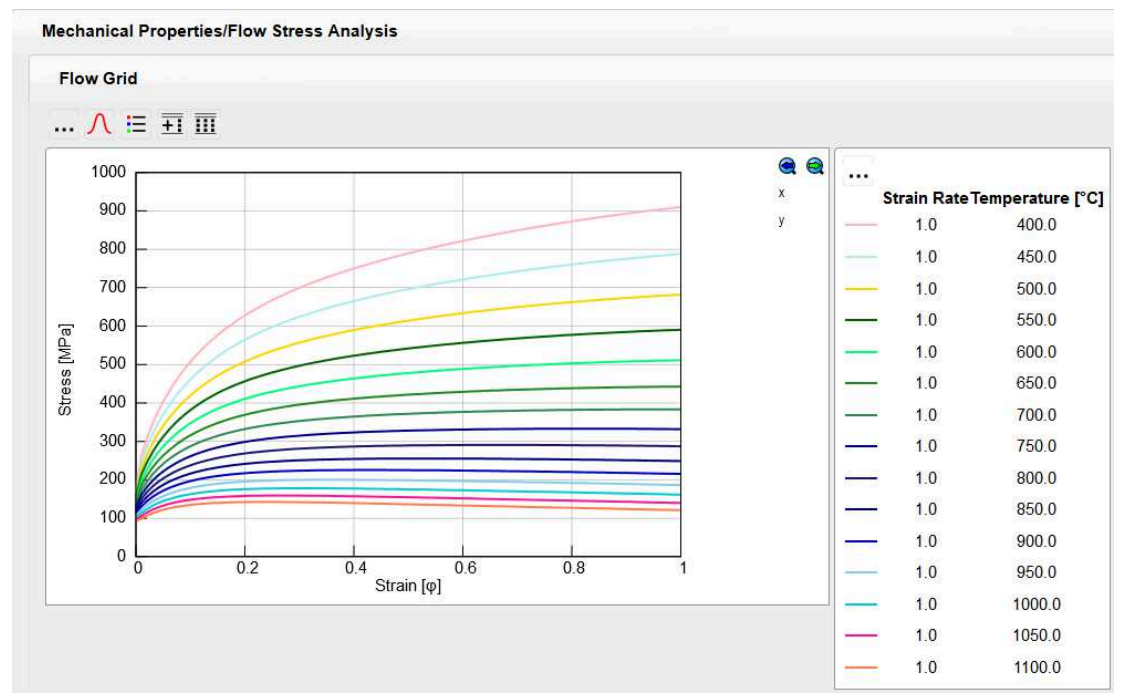

Fig.5 Generated set of flow curves for 22MnB5.

\section{Conclusion}

When presented with the task of generating a consistent model for complex material characterization, various data sources and simulation often exhibit conflicting or insufficient data values. Brute-force fitting of a multi-dimensional model is then a futile endeavour. By splitting the multi-dimensional model in lesser dimensional components and selectively choosing relevant data along each coordinates, one can incrementally construct an unbiased and physically consistent multi-dimensional function. Our commercially available scientific data environment MatPlus allows not only storage and visualization of heterogeneous data, but also such complex manipulation and mathematical modeling, all within a user-friendly interface.

\section{References}

[1] Sente Software Ltd. JMatPro: Practical Software for Materials Properties

[2] Spittel, M., Spittel, T. (2009). 2 Flow stress of steel, in Metal Forming Data of Ferrous Alloys deformation behaviour, pp.23-56

[3] Hochholdinger, B. (2012). Simulation des Presshärteprozesses und Vorhersage der mechanischen Bauteileigenschaften nach dem Härten.

[4] Naderi, M., Molinari, A., Durrenberger, L., \& Bleck, W. (2008). Constitutive relationships for $22 \mathrm{MnB} 5$ boron steel deformed isothermally at high temperatures. Materials Science and Engineering A, 478, pp. 130-139. 